\title{
Imaging of selected sources from the Methanol Multibeam Survey
}

\author{
D. Wong-McSweeney ${ }^{1}$, G. A. Fuller, S. Etoka
}

School of Physics and Astronomy, University of Manchester, Sackville Street Building, Sackville Street, PO Box 88, Manchester, M60 1QD

${ }^{1}$ email: d.wong-mcsweeney@postgrad.manchester.ac.uk

\begin{abstract}
High angular resolution observations are essential for understanding the nature of maser emission and the sources which excite it. Here we present preliminary results from MERLIN observations of three methanol masers from the Torun survey. These MERLIN observations are being analysed as part of the interferometric component of the Methanol Multibeam (MMB) Survey which is surveying the Galactic plane at $|b| \leqslant 2^{\circ}$ for $6.67 \mathrm{GHz}$ methanol maser sources.
\end{abstract}

Keywords. masers, surveys, stars: formation, ISM: individual (G20.24+0.07, G22.45-0.17, G23.01-0.41) galaxies: individual (Milky Way)

\section{Introduction}

The process of high mass star formation is poorly understood relative to that for low mass stars. The $6.67 \mathrm{GHz}$ methanol maser is known to be associated exclusively with regions of massive star formation and therefore searching for this maser will aid in the location and further study of these regions. The Galactic Methanol Multibeam Survey (MMB, Green et al. 2007) aims to find all (to a noise level of $\sim 0.2 \mathrm{Jy}$ ) of the $6.67 \mathrm{GHz}$ methanol masers in the Galactic plane in the range $|b| \leqslant 2^{\circ}$. In parallel with single dish observations made using the Parkes telescope and the Lovell telescope at Jodrell Bank, interferometer observations using the ATCA and MERLIN are being used to determine source positions more accurately. An earlier methanol maser survey (Szymczak et al. 2002), using the 32 $\mathrm{m}$ dish at Torun in Poland, searched the region $20^{\circ} \leqslant l \leqslant 40^{\circ},|b| \leqslant 0^{\circ} .52$ of the Galactic plane, an area that is being covered by the MMB survey. Of the 100 sources found in the Torun survey, 60 needed interferometric follow up,of which 44 have now been observed. The preliminary results of the analysis of three sources, G20.24+0.07, G22.45 - 0.17, and G23.01 - 0.41 from both archive and new MERLIN observations, are presented here. The high angular resolution of these observations $\left(\sim 0.04^{\prime \prime}\right)$ allows the reliable association of the masers with near and mid IR sources. The aim of this project is to determine the properties of these sources and the relationship between different maser species.

\section{Sources and results}

All three sources show complex velocity structure in their methanol spectra. G22.450.17 , with the most complex velocity structure, has eight velocity components whose peaks are separated by $\sim 2 \mathrm{~km} \mathrm{~s}^{-1}$, and spread over a range of $\sim 18 \mathrm{~km} \mathrm{~s}^{-1}$. These features correspond to four spatial components (Figure 1). G23.01-0.41, conversely, has a more simple velocity structure and a more complex spatial structure. G20.24+0.07 has been resolved from a single source in the Torun survey, into two sources separated by $\sim 8.6^{\prime \prime}$ in the north-east south-west direction, and $\sim 1 \mathrm{~km} \mathrm{~s}^{-1}$ in velocity.

The sources are at near/far distances of $6 / \sim 12,5 / 12.8$ and 2.7/15.8 kpc for G20.24 +0.07 , G22.45-0.17, and G23.01-0.41 respectively (Caswell \& Haynes 1983). 

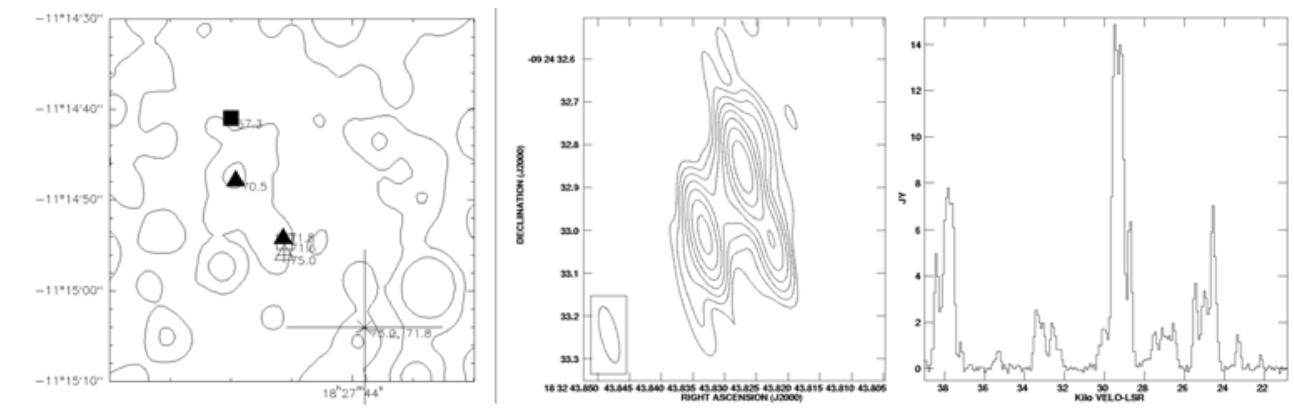

Figure 1. Left: G20.24+0.07 region, RA/Dec in J2000, Grey and contours - 2MASS K-band $2.2 \mu \mathrm{m}$. Masers: $\Delta$ MERLIN $6.67 \mathrm{GHz} \mathrm{CH}_{3} \mathrm{OH} ; \mathbf{\square} 22 \mathrm{GHz} \mathrm{H} \mathrm{O}_{2} ; 1720 \mathrm{MHz} \mathrm{OH} ; \square 6030 / 6035$ $\mathrm{MHz} \mathrm{OH}$; 3 line cross 4660/4750/4765 MHz OH; Diagonal Cross $12 \mathrm{GHz} \mathrm{CH}_{3} \mathrm{OH}$; The size of the '+'s indicate the position accuracy. Velocities, in $\mathrm{km} \mathrm{s}^{-1}$, are marked where known. Refs: Caswell et al. 1995, Caswell et al. 2000, Caswell 2003, Smitts 2003, Szymczak \& Gérard 2004, Testi et al. 1998. Middle: G22.45-0.17 MERLIN 6.67 GHz integrated intensity map with beam shape in the lower left. Right: G22.45-0.17 MERLIN spectrum with velocity in $\mathrm{km} \mathrm{s}^{-1}$.

Both G22.45-0.17 and G23.01-0.41 are seen in the $\mathrm{J}=2-1$ transition of $\mathrm{SiO}$, which is suggestive of shocked material (Harju et al. 1998) as $\mathrm{SiO}$ is usually found on grains rather than as a gas. Of the three sources only G20.24+0.07 is coincident with K-band, $2.2 \mu \mathrm{m}$ 2MASS emission, and it is also found to have emission from $\mathrm{C}^{32} \mathrm{~S}(\mathrm{~J}=2-1)$ which is a tracer of dense gas. The $\mathrm{C}^{32} \mathrm{~S}$ is observed at a velocity of $71.9 \mathrm{~km} \mathrm{~s}^{-1}$ which is comparable to the other maser species in the region with the exception of the $22 \mathrm{GHz}$ water maser (Szymczak et al. 2005) suggesting that this traces different material, for example an outflow. Only G20.24+0.07 is associated with an IRAS point source (IRAS 18249-1116), which has a luminosity of $2.8 \times 10^{4} L_{\odot}$ (Larionov et al. 1999).

\section{Acknowledgements}

Thanks to MMB collaborators: Jim Cohen, Phil Diamond, Malcolm Gray, Jimi Green, Lyshia Quinn, Manchester; Antonio Chrysostomou, Michele Pestalozzi, Mark Thompson, Hertfordshire; Melvin Hoare, Leeds; Mike Masheder, Bristol; Derek Ward-Thompson, James Cox, Cardiff; Jeremy Yates, University College, London; James Caswell, Kate Brooks, Naomi McClure-Griffiths, Maxim Voronkov, Chris Phillips, ATNF, CSIRO; Michael Burton, New South Wales; Andrew Walsh, James Cook; Simon Ellingsen, Tasmania.

\section{References}

J. L. Caswell \& R. F. Haynes 1983, Aus.J.Phy 36, 417

J. L. Caswell, R. A. Vaile, S. P. Ellingsen \& R. P. Norris 1995, MNRAS 274, 1126

J. L.Caswell, Jiynue Yi, R. S. Booth, \& D. M.Cragg 2000, MNRAS 313, 599

J. L. Caswell 2003, MNRAS 341, 551

J. Green, R. J. Cohen, J. L. Caswell, G. A. Fuller et al. 2007, This volume

J. Harju, K. Lehtinen, R. S. Booth, \& I. Zinchenko 1998, A\& A 132, 211

G. M. Larionov, I. E. Val'tts, A. Winnberg, L. E. B. Johansson, et al. 1999, A\&\&A 139, 257

D. P. Smitts 2003, MNRAS 339, 1

M.Szymczak, A. J. Kus, G. Hrynek, A. Kepa \& E. Pazderski 2002, A\&̧A 392, 277

M. Szymczak \& E. Gérard 2004, A\& A 414, 235

M. Szymczak, T. Pillai, \& K. M. Menten 2005, A $\& A 434,613$

L. Testi, M. Felli, P. Persi, \& M. Roth 1998, A\&A 129, 495 